\title{
PERCEPÇÃO DE PACIENTES COM DIABETES SOBRE O AUTOCUIDADO
}

\author{
Diabetic patients' perception of self-care \\ Percepción de pacientes con diabetes sobre al autocuidado
}

\author{
Soraya da Silva Trajano (OrclD) \\ Universidade de Fortaleza - UNIFOR - Fortaleza (CE) - Brasil
}

Luiza Valeska de Mesquita Martins (OrclD)

Universidade de Fortaleza - UNIFOR - Fortaleza (CE) - Brasil

Tallys Newton Fernandes de Matos (OrclD)

Universidade de Fortaleza - UNIFOR - Fortaleza (CE) - Brasil

Cristina de Santiago Viana Falcão (Lattes)

Universidade de Fortaleza - UNIFOR - Fortaleza (CE) - Brasil

Leila Maria Machado Bezerra (Lattes)

Universidade de Fortaleza - UNIFOR - Fortaleza (CE) - Brasil

Ana Paula Vasconcellos Abdon (OrcID)

Universidade de Fortaleza - UNIFOR - Fortaleza (CE) - Brasil

Ana Maria Fontenelle Catrib (Lattes)

Universidade de Fortaleza - UNIFOR - Fortaleza (CE) - Brasil

\section{RESUMO}

Objetivo: Analisar a percepção de pacientes com diabetes mellitus sobre o autocuidado. Métodos: Estudo qualitativo, realizado em um centro de referência em diabetes e hipertensão em Fortaleza, Ceará, Brasil, no período de fevereiro a junho de 2016. A amostra foi composta por 12 pacientes com diagnóstico de diabetes mellitus do tipo 2 , que frequentavam os encontros do Programa de Responsabilidade Social. Para coleta de dados, realizou-se grupo focal, seguido da análise temática, emergindo quatro categorias: aquisição de hábitos de vida saudáveis; alimentação e atividade física aliadas ao controle do diabetes; uso contínuo de medicamentos na prevenção de agravos; dia a dia nos cuidados com a prevenção do pé diabético. Resultados: Pode-se compreender, por meio dos relatos, que há um desenvolvimento da conscientização embasada na prática de educação e saúde apresentada pelos participantes vinculados ao programa. Os pacientes expressavam para o grupo a importância da sua conscientização em relação aos cuidados essenciais para o controle da diabetes, destacando boas práticas de saúde, tais como: uma alimentação balanceada, a prática regular de exercícios físicos, o não abandono do tratamento medicamentoso e a higienização e o cuidado com os pés. Conclusão: Constatou-se que o Programa de Responsabilidade Social proporcionou mudanças nos hábitos de vida, considerando a prática de educação em saúde fundamental na percepção dos pacientes diabéticos.

Descritores: Percepção; Diabetes Mellitus; Educação em Saúde.

\begin{abstract}
Objective: To analyze the diabetic patients' perception of self-care. Methods: Qualitative study, carried out in a diabetes and hypertension referral center in Fortaleza, Ceará, Brazil, in the period from February to June 2016. The sample consisted of 12 patients diagnosed with type 2 diabetes who attended the meetings of the Social Responsibility Program. For data collection, a focus group was carried out, followed by the thematic analysis, thus emerging four categories: acquisition of healthy life habits; eating and physical activity allied to diabetes management; continuous adherence to medication for prevention of injuries; and daily care with diabetic foot prevention. Results: It can be understood, through the reports, that there is development of awareness based on the practice of education and health presented by the participants linked to the program. The patients expressed to the group the importance of their awareness of the essential care procedures for management of diabetes, highlighting good health practices, such as: a balanced diet, the regular practice of physical exercises, non-abandonment of medication therapy, and foot hygiene and care. Conclusion: The study found that the Social Responsibility Program gave rise to changes in life habits, considering the practice of health education as fundamental to the diabetic patients' perception.
\end{abstract}

Descriptors: Perception; Diabetes Mellitus; Health Education. 


\section{RESUMEN}

Objetivo: Analizar la percepción de pacientes con diabetes mellitus sobre el autocuidado. Métodos: Estudio cualitativo realizado en un centro de referencia en diabetes y hipertensión de Fortaleza, Ceará, Brasil en el período entre febrero y junio de 2016 . La muestra fue de 12 pacientes con el diagnostico de diabetes mellitus tipo 2 y que frecuentaban a los encuentros del Programa de Responsabilidad Social. Se realizó grupo focal para la recogida de datos y en seguida el análisis temático emergiendo cuatro categorías: adquisición de hábitos de vida saludables; alimentación y actividad física asociadas con el control de la diabetes; uso continuo de medicaciones para la prevención de agravios; día a día con los cuidados para la prevención del pie diabético. Resultados: A través de los relatos se pudo comprender el desarrollo de la concienciación basada en la práctica de la educación y la salud presentada por los participantes con vínculo en el programa. Los pacientes expresaron para el grupo la importancia de su concienciación sobre los cuidados esenciales para el control de la diabetes, destacando las buenas prácticas de salud, tales como una alimentación balanceada, la práctica regular de ejercicios físicos, el no abandono del tratamiento medicamentoso y la higienización y el cuidado con los pies. Conclusión: Se constató que el Programa de Responsabilidad Social ha proporcionado cambios en los hábitos de vida considerando la práctica de educación en salud fundamental en la percepción de los pacientes diabéticos.

Descriptores: Percepción; Diabetes Mellitus; Educación en Salud.

\section{INTRODUÇÃO}

Segundo a Política Nacional de Promoção da Saúde (PNPS), a promoção da saúde é caracterizada por um conjunto de ações que ressignificam o modo de produzir saúde, seja individualmente, seja em coletividade ${ }^{(1)}$. A PNPS foi instituída pela Portaria MS/GM n 687, de 30 de março de 2006, e confirmou o compromisso do Estado brasileiro com a ampliação e a qualificação de ações de promoção da saúde através do Sistema Único de Saúde (SUS); sendo adicionada na agenda gestores do SUS e nos Planos Nacionais de Saúde seguintes, expandindo as perspectivas das políticas públicas existentes ${ }^{(1)}$. Sendo assim, a PNPS compromete-se, através dos princípios da autonomia e empoderamento, com o fornecimento de subsídios para que os sujeitos envolvidos possam ser corresponsáveis pela sua saúde ${ }^{(1)}$.

Dessa forma, as ações de promoção da saúde consistem nas principais estratégias para o enfrentamento das doenças crônicas não transmissíveis (DCNT), dentre elas, a diabetes mellitus (DM) ${ }^{(2)}$. Essa doença se destaca pela alta mortalidade, morbidade e por ser fator de risco para patologias do sistema cardiovascular. Acredita-se que haja aproximadamente 415 milhões de indivíduos no mundo com DM, dos quais aproximadamente 193 milhões desconhecem possuir a doença. Estima-se que, em 2040, haverá 642 milhões de pessoas com diabetes ${ }^{(3)}$.

No Brasil, o número de pessoas diagnosticadas com diabetes cresceu $61,8 \%$ em uma década, verificandose que as mulheres são mais acometidas. Dentre as capitais do Brasil, Rio de Janeiro e Boa Vista obtiveram a maior (10,4\%) e menor (5,3\%) prevalência de casos, respectivamente. Enquanto isso, Fortaleza, capital do Ceará, apresentou prevalência de 8,2 casos diagnosticados. Nota-se também que essa enfermidade está mais presente na faixa etária de 65 anos ou mais e com menor escolaridade. Fato que se justifica devido ao crescente aumento do envelhecimento populacional e a baixa escolaridade associada ao menor poder aquisitivo(4).

Verifica-se aumento de casos diagnosticados de obesidade e hipertensão em consequência do aumento de pessoas com diabetes, ou seja, está acontecendo uma epidemia de Doenças Crônicas não Transmissíveis (DCNT) na população, em grande parte, devido aos maus hábitos de estilo de vida, ao sedentarismo e à falta de educação preventiva $^{(5)}$.

Dessa forma, os pacientes com diabetes e seus familiares devem participar de programas de educação nutricional desde a descoberta da doença, com discussão sobre a importância do autocuidado, tornando-o independente quanto a decisões e atitudes relacionadas à alimentação e ao controle metabólico. Por meio desse conhecimento, o paciente poderá conhecer a influência dos alimentos sobre sua glicemia, assim como atuar na prevenção de complicações ${ }^{(6,7)}$.

O DM conceitua-se por síndrome de etiologia múltipla, decorrente da ausência total ou parcial de insulina e/ ou da incapacidade desse hormônio exercer adequadamente seu efeito no organismo. Apresenta comorbidades associadas com complicações agudas ou crônicas e a ligação com o tempo de diagnóstico por hiperglicemia crônica acompanhada de alterações metabólicas ${ }^{(3,8,9)}$.

Durante a monitorização dos pacientes com diabetes, percebe-se uma baixa adesão ao tratamento, o que se torna um desafio para os profissionais de saúde, que buscam estabelecer estratégias para diminuir essa ocorrência. 
Nessa perspectiva, a adesão ao tratamento corresponde a seguir as recomendações dos profissionais de saúde e adotar comportamentos saudáveis ${ }^{(10)}$.

Nesse aspecto, a educação em saúde é considerada uma estratégia importante para a promoção da saúde dos pacientes com diabetes através do estímulo ao autocuidado, pois desenvolve a conscientização sobre a doença e torna os envolvidos protagonistas dos cuidados que devem adotar para que obtenham o controle e a redução das complicações. O paciente ganha autonomia para que, sozinho, possa executar hábitos de vida saudáveis em sua rotina, contribuindo para a manutenção do seu bem-estar ${ }^{(111)}$. Nesse sentido, a relevância deste estudo deve-se a importância quanto à investigação da apreensão do conhecimento sobre o autocuidado de pacientes com diagnóstico de diabetes que participam de um programa de educação em saúde. Os resultados contribuirão par o fortalecimento da promoção da saúde com enfoque no autocuidado em pacientes com diabetes para a proposição de estratégias educativas e a consequente redução das comorbidades decorrentes da doença

Portanto, objetivou-se analisar a percepção de pacientes com diabetes mellitus sobre o autocuidado.

\section{MÉTODOS}

Realizou-se um estudo qualitativo ${ }^{(12)}$ no Centro Integrado de Diabetes e Hipertensão (CIDH), unidade da Secretaria da Saúde do Estado do Ceará, localizada na cidade de Fortaleza, Ceará, Brasil, no período de fevereiro a junho de 2016. O CIDH faz parte da rede de atenção de nível secundário e é referência na prevenção e tratamento de pacientes com diabetes e hipertensão que residem na capital e no interior do estado.

Participaram 12 pacientes com diagnóstico de diabetes tipo 2, que frequentavam assiduamente os encontros do Programa de Responsabilidade Social "Despertar Saúde: Mente, Corpo e Movimento - perspectivas em torno de um envelhecimento ativo".

O Programa de Responsabilidade Social acontece desde 2006 e se trata de um grupo aberto aos pacientes que recebem atendimento no setor de Fisioterapia do $\mathrm{CIDH}$, quinzenalmente, por meio de dinâmicas de grupo, rodas de conversa, oficinas e exposições orais. É coordenado pela fisioterapeuta da instituição e tem a participação de estudantes dos cursos de Fisioterapia, Farmácia e Psicologia de uma universidade particular do mesmo município, além de convidados que articulam o tema de cada encontro.

Em caráter multidisciplinar, participaram dos encontros profissionais convidados das áreas de Fisioterapia, Farmácia, Psicologia e Enfermagem, durante oito oficinas, tratando das seguintes temáticas: atividade física, higiene corporal, adesão aos medicamentos, alimentação, cuidados com o pé e imagem corporal.

Incluíram-se os pacientes com diabetes mellitus do tipo 2, com faixa etária de 59 a 80 anos, que participaram de até $80 \%$ dos encontros do Programa de Responsabilidade Social, sendo exclusos os pacientes com diagnóstico pré-estabelecido de alterações psiquiátricas, além dos ausentes por viagem ou por doença.

Integraram a população em estudo 48 pacientes, encerrando a amostra em 12 participantes, por ser um dos critérios do grupo focal ${ }^{(13)}$, tendo como base as questões norteadoras que contemplaram assuntos abordados durante o Programa de Responsabilidade Social: "De que forma a participação nas reuniões do Programa De Responsabilidade Social do CIDH contribuiu para a mudança de hábitos e mudanças de vida?; Como as reuniões influenciaram no controle da diabetes?; Como considera, em termos de importância, a influência das orientações dos profissionais da saúde, como o fisioterapeuta, o enfermeiro, a nutricionista, psicóloga e o farmacêutico, no controle da diabetes?; A atividade física influencia na melhora da condição física?; A boa higiene corporal tem influência na diabetes?; A alimentação adequada interfere de que forma na diabetes?; Quais os cuidados realizam para o pé diabético?; A adesão ao medicamento permitiu que mudanças na saúde?"

Solicitou-se aos participantes o preenchimento de um questionário sobre perfil sociodemográfico (idade, sexo, estado civil, renda, escolaridade, religião e ocupação). O grupo focal ocorreu em um pequeno auditório reservado, preservando o sigilo e o anonimato dos participantes. Utilizou-se para identificação nesta pesquisa a letra "P", significando participante, e os números de 1 a 12 para classificar a ordem das falas, respeitando os aspectos éticos da pesquisa. As falas foram registradas e filmadas com auxílio de um dispositivo móvel.

As informações foram analisadas através da análise temática ${ }^{(14)}$, que se constituiu de três etapas, sendo a primeira etapa a pré-análise, que é composta da leitura flutuante, escolha dos documentos e formulação de hipóteses e objetivos. A segunda etapa é a exploração do material, e a terceira etapa o tratamento dos resultados obtidos e interpretação. A análise aconteceu também seguindo um modelo teórico em pesquisas com população idosa(15).

Após a análise emergiram quatro categorias: aquisição de hábitos de vida saudáveis; alimentação e atividade física aliadas ao controle da diabetes; uso contínuo de medicamentos na prevenção de agravos; dia a dia nos cuidados com a prevenção do pé diabético. 
Destaca-se que o projeto recebeu aprovação do Comitê de Ética em Pesquisa com Seres Humanos da Universidade de Fortaleza, com Parecer $n^{\circ} 1.666 .684$.

\section{RESULTADOS E DISCUSSÃO}

Para apresentação e discussão dos resultados, optou-se por apresentar os dados referentes ao perfil sociodemográfico dos participantes. Em seguida, é dado o enfoque e detalhamento para cada uma das categorias formadas.

\section{Dados sociodemográficos dos entrevistados}

Dos 12 pacientes que participaram do grupo focal, pode-se afirmar que: oito eram do sexo feminino, 10 eram casados, sete tinham renda mensal de dois a três salários mínimos, sete tinham somente a educação básica, 10 eram católicos, e sete eram aposentados. A faixa etária situou-se entre 59 a 80 anos.

Nos relatos, identificaram-se aspectos do comportamento dos pacientes entrevistados que interferem no cuidado à saúde, através das categorias apresentadas: aquisição de hábitos de vida saudáveis; atividade física e alimentação aliada ao controle da diabetes; uso contínuo de medicamentos na prevenção de agravos; dia a dia nos cuidados com pé diabético.

\section{Aquisição de hábitos de vida saudáveis}

Esta categoria apresenta como o programa interferiu positivamente nas mudanças ocorridas na vida das pessoas com diabetes após participação nos encontros. Ressalta-se que a equipe multiprofissional desta pesquisa estava sempre voltada para a observação da PNPS com seus pacientes. Vale reforçar que, dentre os objetivos específicos da PNPS, está a promoção do empoderamento para a capacidade para tomada de decisão e a autonomia de sujeitos e da coletividade por meio do incremento de aptidões pessoais e de capacidades em promoção e defesa da saúde e da vida(1).

Assim, o programa social apareceu como um fator benéfico, considerando que contribuiu com informações úteis para o aprendizado e, consequentemente, mudanças de hábitos dos participantes. Além disso, em conformidade com a PNPS, promoveu o empoderamento para terem autonomia de suas ações, como observado por meio das falas dos participantes sobre o programa:

"Mudou o nosso tipo de ser, o jeito da gente se alimentar, tem que mudar. Os exercícios que a gente não pode deixar de fazer, só melhorou a vida da gente." (P1)

“(...) Os alimentos, a convivência, cada dia que eu venho aqui eu aprendo muito mais, eu acho muito bom. $E$ sobre as explicações dos medicamentos, os remédios, e de farmácia para mim mudou muito, foi ótimo." (P2)

"É muito importante, porque vai lembrando, vai puxando aquilo que a gente se esquece que o médico fala, não é porque a gente queira (...) Mas é uma forma de puxar, sacudir nosso interesse, nossa participação, pra nossa saúde. É muito importante mesmo. E assim, a gente se conscientiza e muda mesmo." (P3)

Corroborando os achados da presente pesquisa, uma intervenção educativa em diabetes realizado em um Centro de Saúde Escola Estoril, São Paulo, no Brasil, verificou que os pacientes com diabetes que participaram da educação em saúde elevaram o nível de conhecimento sobre a doença em relação àqueles que não participaram, representando a eficácia dessas ações ${ }^{(16)}$.

Outro estudo em Belo Horizonte, Minas Gerais, Brasil, menciona que os pacientes com essa enfermidade, iniciaram o programa educativo com pouco conhecimento e cuidado no controle do DM, porém o envolvimento com o programa possibilitou mudanças comportamentais. Com isso, os participantes com maior tempo de participação tiveram melhores registros nos autocuidados diários ${ }^{(17)}$.

Em relação aos hábitos que os participantes do atual estudo tinham e que mudaram no dia a dia, enfatizaram a alimentação, a aquisição de cuidados pessoais e a atividade física. Essa alteração comportamental vai além do próprio corpo, expandindo para o ambiente físico e domiciliar, em que cada detalhe ganha relevância no novo estilo de vida. Pode-se exemplificar esse pressuposto através das falas:

"Maneira de se alimentar, como se cuidar e cuidar dos pés." (P1)

“Eu comecei a mudar, mas mudei mesmo. A gente aprendeu muito, cortar as unhas dos pés, ter cuidado, limpeza, passar um creme, uma benção mesmo (...) A gente faz as atividades, os exercícios; às vezes, a gente 
se esquece de fazer, mas a gente tenta, viu?!" (P4)

"Da limpeza, cuidar do corpo e também dos alimentos, e também até da casa. Muitas coisas." (P2)

"Eu tenho minha própria toalha para enxugar o pé (...) meu cabelo, eu lavo todo dia." (P8)

Estudo realizado com 16 idosos em Maringá, Paraná, Brasil, destaca a importância das ações educativas. Após essas ações, os pacientes perceberam a importância de cuidar da sua saúde e se sentiram mais estimulados para mudanças de hábito na alimentação e na prática de atividade física ${ }^{(18)}$.

O Programa de Responsabilidade Social desenvolvido no CIDH em Fortaleza, Ceará, Brasil, proporciona aos participantes atividades de prevenção e promoção da saúde. Segundo os participantes, os cuidados com a pele também são necessários, pois ela serve como "barreira" contra microrganismos externos. Nesse aspecto, um estudo realizado com 212 pacientes com diabetes evidenciou que existem três complicações dermatológicas frequentes provocadas pela ausência de cuidados com a pele dos pés: xeroses, onicomicoses e fissuras ${ }^{(19)}$.

\section{Alimentação e atividade física aliadas ao controle do diabetes}

Nesta categoria, os participantes citam os alimentos que consomem, discutem sobre o preço dos alimentos e evidenciam o tempo e a prática de exercícios físicos, principalmente os aeróbicos, destacando como imprescindível para uma boa saúde a combinação da atividade física com uma boa alimentação.

Aqui, nesta temática, buscava-se focar em temas prioritários da PNPS evidenciados pelas ações de promoção da saúde, como alimentação adequada e saudável, que compreende promover ações relativas à alimentação adequada e saudável, visando à promoção da saúde e à segurança alimentar e nutricional(1). Também abordava-se a questão das práticas corporais e atividades físicas, que compreende promover ações, aconselhamento e divulgação de práticas corporais e atividades físicas ${ }^{(1)}$.

Sobre esse assunto, os participantes atribuíram à retirada de alimentos de sua dieta, como o arroz, carne bovina e outros, assim ocorrendo a diminuição da glicose, como exemplificado nas falas:

"Minha diabetes está controlada. A minha glicemia hoje deu 101 e antes dava 250...190... Aí tirei o arroz, dá 96...85... Hoje, deu 101, graças à Deus." (P5)

"A minha está controlada [...]. Eu fui para a doutora [...] e ela disse: O senhor comeu carne de gado? Eu respondi: Não, senhora. Ela: Pois não coma! Coma alface, tomate, folha, peixe, galinha. E minha glicemia fica praticamente controlada. Ontem, minha glicemia deu 73, sempre dá 100 pra baixo." (P6)

Em contrapartida, um estudo realizado em Montes Claros, Minas Gerais, Brasil, mostrou que as orientações dadas pelos profissionais de um programa de educação em saúde eram escassas, pois os pacientes com diabetes ainda apresentavam lacunas quanto ao conhecimento sobre a alimentação adequada, necessitando de um acompanhamento individual(20), evidenciando que o trabalho em grupo é relevante mas que também há necessidade da consulta individual, respeitando a singularidade de cada um.

A ingestão de alimentos saudáveis contribui para diminuição do índice glicêmico e da carga glicêmica global na dieta. Com isso, destaca-se que a maior quantidade de carboidratos na alimentação, como arroz, não proporciona controle glicêmico(21).

Quando questionados sobre a alimentação saudável, citaram como preferências frutas e hortaliças:

"Bem, eu como verdura, fruta e leite de soja." (P2)

Nessa perspectiva, um estudo com 30 idosos com diabetes, em Porteiras, Ceará, Brasil, consta uma realidade oposta de hábitos alimentares, pois, embora tenha constatado o consumo de frutas diário, havia baixa ingestão de hortaliças e sucos naturais ${ }^{(22)}$.

Os alimentos normalmente são elaborados pelos participantes, tendo em vista o alto custo de alimentos industrializados para esses pacientes, como apresentado nas falas abaixo:

“Lá em casa sou eu quem faço." (P4)

"Porque os alimentos para diabetes são muito caros, eu ganho pouco, eu tomo muito remédio. [...] Eu compro quatrocentos reais de remédio todo mês, quase o que eu recebo, então eu me alimento de acordo com meus probleminhas e minhas condições financeiras." (P1) 
Um estudo apontou que os brasileiros de baixa renda apresentam dificuldades de se alimentar corretamente com uma nutrição balanceada, conforme as recomendações nacionais, enquanto que os de média e alta renda gastam grande parte do seu dinheiro com alimentos pouco saudáveis ${ }^{(23)}$.

Outra pesquisa com idosos que frequentavam o Centro de Convivência de Idosos em Sairé, Pernambuco, Brasil, evidencia que o grau de conhecimento sobre alimentação e a DM foi adequado, mas observou-se que aspectos socioeconômicos e culturais podem interferir na adesão ao tratamento nutricional, ou ainda nas preferências alimentares $^{(24)}$.

Ressalta-se a importância do conhecimento dos profissionais de saúde sobre as dificuldades dos usuários e da necessidade de adequação das ações de promoção da alimentação saudável, visto que um estudo realizado em Pelotas, Rio Grande do Sul, Brasil, destacou as dificuldades dos participantes para ter uma alimentação saudável, tais como: o valor alto dos alimentos saudáveis, a pouca força de vontade, a obrigação de ter que evitar alimentos considerados proibidos e a falta de tempo e de conhecimento ${ }^{(25)}$.

Identificou-se a prática da atividade física, como as atividades sociais desenvolvidas pelos bombeiros em equipamentos disponíveis nas praças, e o maior incentivo e divulgação por meio de projetos sociais, citados a seguir:

"Eu faço caminhada, alongamento, brinco, faço tudo que tenho direito e mais alguma coisa." (P5)

"Eu faço exercício naqueles equipamentos que tem na praça." (P3)

"Meu apartamento já é quase uma academia, porque os netos vão lá e a gente pula corda, faz karatê, faz todo tipo de exercício." (P8)

Com isso, os exercícios praticados moderadamente são capazes de melhorar a glicemia e contribuem para a redução de complicações renais. Constatou-se que realizar exercícios físicos por três vezes na semana durante três meses foram suficientes para exibir transformações significativas nos aspectos fisiopatológicos ${ }^{(26)}$.

O tempo de atividade física também foi mencionado nas falas por meio da satisfação com a inclusão do exercício no dia a dia, e somente uma participante relatou não fazer caminhada por conta das dores no joelho.

"Já faz mais ou menos três a quatro anos que pratico atividade física." (P1)

“(..) Tem mais de 30 anos que faço atividade física." (P8)

"Está com 15 anos ou mais que eu faço atividade física, faço caminhada e faço a bola." (P7)

É necessário que a sociedade se mantenha ativa, minimizando riscos para limitações físicas, pois o exercício físico promove a diminuição do índice glicêmico quando segue orientações sobre a duração, intensidade e resistência adequadas $^{(27)}$.

\section{Uso contínuo de medicamentos na prevenção de agravos}

Nesta categoria, os participantes explicam que são cuidadosos em relação à ingestão da maneira correta de seus remédios. No entanto, situações imprevisíveis, como a falta do remédio nas farmácias, eram motivos que causavam a interrupção do tratamento.

Apurou-se que todos se apresentaram fazendo o uso correto e com o cuidado de tomar no horário indicado, tomando-o pausadamente com intervalo de 10 minutos entre um e outro e, principalmente, com a disciplina de tomar diariamente. Reforçando que os pacientes afirmaram fazer o uso dos seus medicamentos, há a seguinte fala:

"Não só o medicamento, como a insulina também. Nunca suspendi o remédio." (P1)

Contrariando os achados da pesquisa, um estudo com 142 pessoas com DM tipo 2 no interior de Minas Gerais, Brasil, mostrou que 66 (46,5\%) pacientes faziam uso de medicamento às vezes; 49 (34,4\%) pacientes nunca esqueciam; $13(9,1 \%)$ quase sempre esqueciam; sete $(4,9 \%)$ raramente esqueciam; duas $(1,4 \%)$ esqueciam com frequência, e, por fim, cinco $(3,6 \%)$ esqueciam sempre. Portanto, é inevitável o conhecimento sobre os riscos relacionados e a necessidade do uso regular dos medicamentos, bem como sobre o controle das manifestações da doença( ${ }^{(28)}$.

Em contrapartida, outros membros indicaram não tomar corretamente pela falta de remédio nas farmácias, tanto nas privativas como também nas farmácias das unidades básicas. Quanto a isso, destaca-se:

"Meu esposo passou uns dois a três dias sem tomar o medicamento, porque nas farmácias não tinha, e eu andei em várias farmácias e não tinha." (P2)

"Até porque o remédio do diabetes é assim: se a gente tomar direitinho, ela vai controlar; agora se bagunçar, vai baldear. A diabetes descompensa e a pessoa não sabe o porquê." (P1) 
É importante que políticas e projetos atuem como forma de educação contínua, desenvolvendo estratégias essenciais para suprir possíveis irregularidades na distribuição de medicamentos. Nessa lógica, foi realizado um estudo transversal na região Sudeste do Brasil, com 423 entrevistados, em que 357 dos pacientes apresentaram adesão ao tratamento. Com isso, $84,4 \%$ apresentaram adesão ao tratamento medicamentoso, $58,6 \%$ apresentaram adesão ao exercício físico e 3,1\%, ao plano alimentar. Destacou-se que a irregularidade no tratamento possibilita a chance do descontrole glicêmico. Porém pacientes com mais de 10 anos de diagnóstico apresentaram maior adesão ao tratamento medicamentoso, alimentar e exercício físico. Portanto, há um nível maior de conhecimento e conscientização nesses indivíduos, e na ausência de um componente do tratamento são elaboradas estratégias para suprir o componente em falta, como alimentação e exercício físico ${ }^{(29)}$.

\section{Dia a dia nos cuidados com a prevenção do pé diabético}

Nessa categoria, os pacientes demonstraram várias formas de cuidar da saúde dos seus pés, inclusive ressaltaram a participação dos familiares na colaboração para realização desse cuidado.

Sobre esse tema, os participantes evidenciaram tomar as atitudes necessárias para a prevenção de agravos, sendo participativos em relatar suas experiências no dia a dia quanto aos cuidados, como nas falas:

"Eu peço minha nora pra fazer, porque meu corpo não alcança meus pés. Eu não lixo, eu não corto, até pra lavar eu boto assim (mostrando que trepa o pé em uma cadeira)." (P1)

"Passo a escova, a lixa, aquela mais fina que tem, mas assim, pra mim mesma, eu não consigo. E no dia que ela não está (nora), eu pego a escovinha, lavo a unha ali, enxugo, trepo o pé em algum canto, porque meu corpo não dá." (P1)

"Eu lavo, hidrato, eu corto a unha, todo dia." (P8)

"Eu limpo, passo a escova, passo a lixa para tirar aquela crosta." (P4)

"Não pode passar creme entre os dedos. Cria micose." (P1)

Destaca-se a necessidade do apoio familiar e de amigos com a pessoa que tem diabetes, pois essa participação ajuda tanto nos aspectos emocionais como também no gerenciamento dos devidos cuidados para que se tenha 0 controle dos níveis glicêmicos no sangue ${ }^{(30)}$.

Esses cuidados são essenciais na prevenção de lesões do pé dos diabéticos, porém, um estudo sobre o autocuidado com os pés desenvolvido com 331 portadores de diabetes tipo 2 apresentou que somente 128 examinavam os pés, 96 examinavam os sapatos antes de calçá-los e 135 secavam os espaços interdigitais depois de lavar os pés de 5 a 7 dias na semana. Tais atitudes, quando realizadas diariamente, diminuem a chance de complicações ${ }^{(31)}$.

O presente estudo confirma que a adoção de programas de educação em saúde apresenta resultados benéficos, pois revela uma realidade diferente daquela que é retratada nos indicadores epidemiológicos. Os participantes puderam perceber a importância de um planejamento alimentar balanceado, o qual deve ser prioridade em todos os programas voltados a indivíduos com $\mathrm{DM}^{(6)}$. Dessa forma, reforça-se que as estratégias de educação em saúde abrangem ações em grupos operativos, oficinas e palestras. A alimentação está diretamente relacionada a questões psicossociais e culturais, portanto é relevante introduzir tais considerações no processo educativo(9).

Como possibilidade e estratégia para subsidiar planos de ação, pôde-se compreender, ao longo da pesquisa, que a relação entre educação e saúde é imprescindível, desde a baixa escolaridade até o diagnóstico e tratamento. Nesse sentido, a educação é um elemento chave para a modificação desse contexto, buscando o desenvolvimento e transformação na saúde. Com base nesse programa, pôde-se identificar e elaborar um plano percebido como essencial ao tratamento de pacientes com diabetes. Esse plano se configura em quatro momentos essenciais em assimilação com os quatro pilares da educação ${ }^{(32)}$ para a reconfiguração da saúde do paciente.

No primeiro momento, destaca-se a transmissão do "conhecimento" como elemento fundamental para a superação na ausência de educação em saúde. Nesse momento, é importante que se configure a motivação, levando em consideração a possibilidade de descontinuidade no tratamento do paciente. Assim, são repassadas todas as informações sobre a doença e o tratamento referente ao movimento de integração do modelo biopsicossocial.

No segundo momento, destaca-se a "prática" através dos conhecimentos obtidos, a fim de modificar o cenário da doença, reconfigurando a busca pela promoção da saúde. Neste, os pacientes praticam as orientações transmitidas, iniciando mudanças comportamentais com a finalidade da prevenção e promoção da saúde.

No terceiro momento, identificou-se a "convivência" com o processo estabelecido anteriormente, elaborando a conscientização sob as novas atitudes que possibilitam saúde. Isto proporciona autoestima, motivação e novos 
estímulos no cuidado com a saúde. Percebem-se essas características através do raciocínio na busca e continuidade do tratamento por meio de estratégias no enfrentamento de dificuldades adversas.

No quarto momento, detecta-se o movimento constante e integrativo dos momentos citados anteriormente na busca pela atualização e desenvolvimento do "ser". Esse processo mostra-se contínuo, no qual o indivíduo busca a transformação significativa dos fatores percebidos como inacabados. Com isso, destaca-se que esses processos possibilitarão, significativamente, benefícios para a saúde dos pacientes.

Por fim, o presente estudo apresentou limitações, tais como a dificuldade do pesquisador em criar vínculo com os participantes, devido ausência nos encontros quinzenais que ocorreram previamente à coleta dos dados, e a dificuldade em garantir a participação de todos os pacientes que fizeram parte do grupo focal.

Diante do exposto, orienta-se que outros serviços de saúde, como as Unidades Básicas de Saúde, promovam orientações sobre o autocuidado em diabetes rotineiramente, visto que facilita a continuidade do tratamento no âmbito domiciliar, favorecendo a integralidade do cuidado.

\section{CONSIDERAÇÕES FINAIS}

O estudo constatou que o Programa de Responsabilidade Social proporcionou mudanças nos hábitos de vida, considerando a prática de educação em saúde fundamental na percepção dos pacientes diabéticos.

Os encontros possibilitaram mudanças de hábitos relacionados à adesão a uma alimentação balanceada com frutas e verduras, à prática regular de exercício físico, à ingestão de medicamentos de modo contínuo e à manutenção dos cuidados com os pés, buscando prevenir a formação de úlceras.

Dessa forma, os participantes do estudo se apresentaram conscientes da importância da prática de atividade física, da alimentação adequada, do uso contínuo dos medicamentos e dos cuidados que devem adotar a fim de evitar amputações do pé.

\section{AGRADECIMENTOS}

Ao Centro Integrado de Diabetes e Hipertensão e à Secretaria da Saúde do Estado do Ceará.

\section{CONTRIBUIÇÕES}

Soraya da Silva Trajano, Cristina de Santiago Viana Falcão, Leila Maria Machado Bezerra, Ana Maria Fontenelle Catrib: concepção e delineamento do estudo; análise e interpretação dos dados; redação e aprovação final da versão a ser publicada. Luiza Valeska de Mesquita Martins, Tallys Newton Fernandes de Matos: análise e interpretação dos dados; redação do manuscrito e aprovação final da versão a ser publicada. Ana Paula Vasconcellos Abdon, redação do manuscrito e aprovação final da versão a ser publicada.

\section{REFERÊNCIAS}

1. Ministério da Saúde (BR). Política Nacional de Promoção da Saúde: PNPS: revisão da Portaria MS/GM nº 687 , de 30 de março de 2006. Brasília: Ministério da Saúde; 2015.

2. Malta DC, Silva JB Jr. Plano de Ações Estratégicas para o Enfrentamento das Doenças Crônicas Não Transmissíveis no Brasil após três anos de implantação, 2011-2013. Epidemiol Serv Saúde. 2014;23(3):389-98.

3. Diabetesatlas.org. IDF diabetes atlas - Home [Internet]. 2018 [acesso em 2018 Fev 17]. Disponível em: http:// www.diabetesatlas.org/

4. Ministério da Saúde (BR). Vigitel Brasil 2016: vigilância de fatores de risco e proteção para doenças crônicas por inquérito telefônico. Brasília: Ministério da Saúde; 2017.

5. Oliveira JEP, Vencio S. Diretrizes da Sociedade Brasileira de Diabetes (2015-2016). São Paulo: A.C. Farmacêutica; 2016.

6. Santos RD, Gagliardi ACM, Xavier HT, Magnoni CD, Cassani R, Lottenberg AMP, et al. I Diretriz sobre o Consumo de Gorduras e Saúde Cardiovascular. Arq Bras Cardiol. 2013;100(1 Supl 3):1-40.

7. Funnel MM, Brown TL, Childs BP, Haas LB, Hosey GM, Jensen B, et al. National standards for diabetes selfmanagement education. Diabetes Care. 2010;33(Suppl 1):S89-96. 
8. American Diabetes Association. Standards of Medical Care in Diabetes. Diabetes Care. 2013;36(1):11-66.

9. Boell J, Ribeiro R, Silva D. Fatores de risco para o desencadeamento do pé diabético. Rev Eletrônica Enferm. 2014;16(2):386-93.

10. Faria HTG, Santos MA, Arrelias CCA, Rodrigues FFL, Gonela JT, Souza CR, et al. Adesão ao tratamento em diabetes mellitus em unidades da Estratégia Saúde da Familía. Rev Esc Enferm USP. 2014;48(2):257-63.

11. Dias EG, Nunes MSL, Barbosa VS, Jorge AS, Campos LM. Comportamentos de pacientes com Diabetes Tipo 2 sob a perspectiva do autocuidado. J Health Sci. 2017;19(2):109-13.

12. Gonçalves $\mathrm{H}$, Menasche R. Pesquisando na interface: problemas e desafios a partir da pesquisa qualitativa. Interface Comun Saúde Educ.2014;18(50):449-56.

13. Gondim SMG. Grupos focais como técnica de investigação qualitativa: desafios metodológicos. Paidéia (Ribeirão Preto). 2002;12(4):149-61.

14. Minayo MCS. O desafio do conhecimento: pesquisa qualitativa em saúde. São Paulo: Hucitec; 2014.

15. Minayo MCS, Coimbra CEA Junior. Antropologia, saúde e envelhecimento. Rio de Janeiro: Editora Fiocruz; 2002.

16. Barbosa L, Borges PCP, Lemos SS, Cesarino CB. Avaliação da intervenção educativa em grupo para diabéticos assistidos em um Centro de Saúde Escola. Rev Enferm UERJ. 2016; 24(2):1-5.

17. Maia MA, Reis IA, Torres HC. Associação do tempo de contato no programa educativo em diabetes mellitus no conhecimento e habilidades de autocuidado. Rev Esc Enferm USP. 2016;50(1):59-65.

18. Motta MDC, Navarro Peternella FM, Santos AL, Teston EF, Marcon SS. Educação em Saúde junto a idosos com hipertensão e diabetes: estudo descritivo. Rev Uningá Review. 2014;18(2):48-53.

19. Lima IG, Costa JFL, Oliveira AF, Borges JN Junior, Peixoto AS, Pancieri MS. Educar para prevenir: a importância da informação no cuidado do pé diabético. Revista Conexão UEPG. 2017; 13(1):186-95.

20. Aquino ACF, Alves BMS, Pinho L. Percepção dos usuários sobre as ações educacionais de alimentação e nutrição no Sistema Único de Saúde. Rev Baiana Saúde Pública. 2016; 40(4):991-1003.

21. Portero-McLellan KC, Srebernich SM, Meireles F, Postali CC, Leandro-Merhi VA. Determinação do índice glicêmico e da carga glicêmica de dietas hospitalares para indivíduos com diabetes. Cad Saúde Colet. 2010; 18(2):259-65.

22. Santos LM, Sampaio JRF, Borba VFC, Luz DCRP, Rocha BEM. Avaliação do hábito alimentar e estado nutricional de idosos com diabetes mellitus tipo 2 atendidos na atenção básica de saúde do município de Porteiras-CE. Rev e-ciência. 2017;5(1):69-77.

23. Borges CA, Claro RM, Martins APB, Villar BS. Quanto custa para as famílias de baixa renda obterem uma dieta saudável no Brasil? Cad Saude Pública. 2015;31(1):137-48.

24. Barbosa MAG, Almeida AMR, Figueiredo MA, Negromonte AG, Silva JSL, Viana MGS, et al. Alimentação e diabetes mellitus: percepção e consumo alimentar de idosos no interior de Pernambuco. Rev Bras Promoç Saúde. 2015;28(3):370-8.

25. Lindemann IL, Oliveira RR, Sassi RAM. Dificuldades para alimentação saudável entre usuários da atenção básica em saúde e fatores associados. Ciênc Saúde Colet. 2016;21(2):599-610.

26. Andrade EA, Fett CA, Vieira RC Junior, Voltarelli FA. Exercício físico de moderada intensidade contribui para o controle de parâmetros glicêmicos e clearance de creatinina em pessoas com diabetes mellitus tipo 2 . Rev Bras Ciênc Mov. 2016;24(1):118-26.

27. Llopis $P Q$, García-Galbis MR. Control glucémico a través del ejercicio físico en pacientes con diabetes mellitus tipo 2; revisión sistemática. Nutr Hosp. 2015; 31(4):1465-72.

28. Rossi VEC, Silva AL, Fonseca GSS. Adesão ao tratamento medicamentoso entre pessoas com diabetes mellitus tipo 2. Rev Enferm Cent Oeste Min. 2015;5(3):1820-30.

29. Faria HTG, Rodrigues FFL, Zanetti ML, Araujo MFM, Damasceno MMC. Factors associated with adherence to treatment of patients with diabetes mellitus. Acta Paul Enferm. 2013;26(3):231-7. 
30. Shawon MS, Hossain FB, Adhikary G, Gupta R, Hashan MR, Rabbi MF, et al. Attitude towards diabetes and social and family support among type 2 diabetes patients attending a tertiary-care hospital in Bangladesh: a crosssectional study. BMC Res Note. 2016;9:286.

31. Rezende DS Neta, Silva ARV, Silva GRF. Adesão das pessoas com diabetes mellitus ao autocuidado com os pés. Rev Bras Enferm. 2015;68(1):111-6.

32. Scherer ZAP, Scherer EA. Identificação dos pilares da educação na disciplina integralidade no cuidado à saúde. Rev Esc Enferm USP. 2012;46(4):985-93.

\section{Endereço para correspondência:}

Soraya da Silva Trajano

Universidade de Fortaleza - UNIFOR

Programa de Pós-Graduação em Saúde Coletiva

Av. Washington Soares, 1321 / Bloco S, Sala 1

Bairro: Edson Queiroz

CEP: 60811-905 - Fortaleza - CE - Brasil

E-mail: soraya_trajano@hotmail.com 